\title{
Research of AUV Shell Mathematics Model
}

\author{
Xiangzhong Meng, Xiuhua Shi, Xiangdang Du \\ College of Marine, Northwestern Polytechnical University, Xi' an 710072, China \\ Tel: 86-29-8847 4122 E-mail: mengxz@mail.nwpu.edu.cn \\ Qinglu Hao \\ Department of Aircraft Structure Overhaul, AMECO Beijing, Beijing 100621, China
}

The research is supported by graduate starting seed foundation of Northwestern Polytechnical University. No. Z200510

\begin{abstract}
The finite element analysis and research on the vibration mode of a certain AUV with the analytic method are expatiated Based on the basic equations of thin shells theory, this paper analyses and sets up the cylindrical shell vibration mathematical model, and validated the correctness of the model by ANSYS. The method presented is effective in analyse and dynamical design of AUV weaken vibration and low noise structure.
\end{abstract}

Keywords: Cylindrical shell, Mathematics model, Vibration mode, AUV, FEA

During the work progress of AUV, such as torpedo and mine, the power system activates vibration of the shell and causes noise radiation in hydro-medium. The radiating noise makes great affect on the concealment, and enlarges the enemy forewarning distance. The research of vibration has important theory value and practical meaning on AUV. The AUV is combines in spherical shell, cylindrical shell, conical shell and other revolutionary shells. The cylindrical shell is the primary, and the spherical shell and the conical shell are similar to the cylindrical shell, so the vibration mode of cylindrical shell is prevalent. This paper provides a method to calculate the eigenfrequency so as to validate the structure plan.

\section{The balance differential equations of free vibration}

A middle surface patch of cylindrical shell and internal forces in the orthogonal coordinate system are shown in Figure 1 $\alpha$ and $\beta$ are the lines of main curvature, and $\gamma$ is the normal pointed to convex direction. $N_{1}, N_{2}, N_{12}, N_{21}, M_{1}$ $M_{2}, M_{12}, M_{21}, Q_{1}, Q_{2}$ are the internal forces acted on the $\alpha$ plane and the $\beta$ plane, $k_{1}$ and $k_{2}$ are the main curvatures on the $\alpha$ direction and the $\beta$ direction, $R$ is the radius of the middle surface, and $k_{1}=0, k_{2}=R$, A and $\mathrm{B}$ are the Lame coefficients on the $\alpha$ direction and the $\beta$ direction, and $A=B=1, p_{1}, p_{2}, p_{3}$ are the component of loads on the $\alpha$ direction, the $\beta$ direction and the $\gamma$ direction. The plus directions of the internal forces are shown in Figure 2.

The sum of all the internal forces components on the $\alpha$ direction divided by $d \alpha d \beta$ is 0 , that is $\sum F_{\alpha}=0$, in the same way, $\sum F_{\beta}=0, \sum F_{\gamma}=0$. The moment of all the internal forces to the $\alpha$ axis is 0 , that is $\sum M_{\alpha}=0$, in the same way, $\sum M_{\beta}=0, \sum M_{\gamma}=0$. After operated, the balance equations of the cylindrical shell are:

$$
\left.\begin{array}{l}
\frac{\partial N_{1}}{\partial \alpha}+\frac{\partial N_{12}}{\partial \beta}+p_{1}=0 \\
\frac{\partial N_{2}}{\partial \beta}+\frac{\partial N_{12}}{\partial \alpha}+\frac{Q_{2}}{R}+p_{2}=0 \\
\frac{\partial Q_{1}}{\partial \alpha}+\frac{\partial Q_{2}}{\partial \beta}-\frac{N_{2}}{R}+p_{3}=0 \\
\frac{\partial M_{1}}{\partial \alpha}+\frac{\partial M_{12}}{\partial \beta}-Q_{1}=0 \\
\frac{\partial M_{2}}{\partial \beta}+\frac{\partial M_{12}}{\partial \alpha}-Q_{2}=0
\end{array}\right\}
$$


From the last two equations of equations (1.1), the expressions of $Q_{1}$ and $Q_{2}$ can be reason out. The expressions reckoned in the former three equations and the affects of the cross shearing force $Q_{2}$ to the balance on the $\alpha$ direction and the $\beta$ direction are neglected, then

$$
\left.\begin{array}{l}
\frac{\partial N_{1}}{\partial \alpha}+\frac{\partial N_{12}}{\partial \beta}+p_{1}=0 \\
\frac{\partial N_{2}}{\partial \beta}+\frac{\partial N_{12}}{\partial \alpha}+p_{2}=0 \\
\frac{\partial^{2} M_{1}}{\partial \alpha^{2}}+2 \frac{\partial^{2} M_{12}}{\partial \alpha \partial \beta}+\frac{\partial^{2} M_{2}}{\partial \beta^{2}}-\frac{N_{2}}{R}+p_{3}=0
\end{array}\right\}
$$

At the state of free vibration, according to D'alembert's Principle, the free vibration balance differential equations of the cylindrical shell are:

$$
\left.\begin{array}{l}
\frac{\partial N_{1}}{\partial \alpha}+\frac{\partial N_{12}}{\partial \beta}+p_{1}-m \frac{\partial^{2} u}{\partial t^{2}}=0 \\
\frac{\partial N_{2}}{\partial \beta}+\frac{\partial N_{12}}{\partial \alpha}+p_{2}-m \frac{\partial^{2} v}{\partial t^{2}}=0 \\
\frac{\partial^{2} M_{1}}{\partial \alpha^{2}}+2 \frac{\partial^{2} M_{12}}{\partial \alpha \partial \beta}+\frac{\partial^{2} M_{2}}{\partial \beta^{2}}-\frac{N_{2}}{R}+p_{3}-m \frac{\partial^{2} w}{\partial t^{2}}=0
\end{array}\right\}
$$

Where, $m$ is the unit area mass of the cylindrical shell middle surface, $u, v$ and $w$ are the component of displacements on the $\alpha$ direction, the $\beta$ direction and the $\gamma$ direction of any point on the middle surface.

The geometric equations (1.4) and the physical equations (1.5) of the cylindrical shell are:

$$
\left.\begin{array}{c}
\varepsilon_{1}=\frac{\partial u}{\partial \alpha} \quad \varepsilon_{2}=\frac{\partial v}{\partial \beta}+\frac{w}{R} \quad \varepsilon_{12}=\frac{\partial u}{\partial \beta}+\frac{\partial v}{\partial \alpha} \\
\chi_{1}=-\frac{\partial^{2} w}{\partial \alpha^{2}} \quad \chi_{2}=-\frac{\partial^{2} w}{\partial \beta^{2}} \quad \chi_{12}=-\frac{\partial^{2} w}{\partial \alpha \partial \beta}
\end{array}\right\}
$$

Where, $\varepsilon_{1}, \varepsilon_{2}, \varepsilon_{12}$ are the inplane strains on the middle surface, $\chi_{1}, \chi_{2}, \chi_{12}$ are the bending strains on the middle surface $E$ is the elastic modulus, $\mu$ is the Poisson's ratio, $D$ is the bending strength, and $D=E h^{3} / 12\left(1-\mu^{2}\right)$.

The strains are eliminated by combining the equations (1.4) and the equations (1.5), the result are brought into the equations (1.5), then, the free vibration balance differential equations of the cylindrical shell expressed by displacements are:

$$
\left.\begin{array}{l}
\frac{\partial^{2} u}{\partial \alpha^{2}}+\frac{1-\mu}{2} \frac{\partial^{2} u}{\partial \beta^{2}}+\frac{1+\mu}{2} \frac{\partial^{2} v}{\partial \alpha \partial \beta}+\frac{\mu}{R} \frac{\partial w}{\partial \alpha}+\frac{1-\mu^{2}}{E h}\left(p_{1}-m \frac{\partial^{2} u}{\partial t^{2}}\right)=0 \\
\frac{\partial^{2} v}{\partial \beta^{2}}+\frac{1-\mu}{2} \frac{\partial^{2} v}{\partial \alpha^{2}}+\frac{1+\mu}{2} \frac{\partial^{2} u}{\partial \alpha \partial \beta}+\frac{1}{R} \frac{\partial w}{\partial \beta}+\frac{1-\mu^{2}}{E h}\left(p_{2}-m \frac{\partial^{2} v}{\partial t^{2}}\right)=0 \\
\frac{h^{2}}{12} \nabla^{2} \nabla^{2} w+\frac{\mu}{R} \frac{\partial u}{\partial \alpha}+\frac{1}{R} \frac{\partial v}{\partial \beta}+\frac{w}{R^{2}}+\frac{1-\mu^{2}}{E h}\left(p_{3}-m \frac{\partial^{2} w}{\partial t^{2}}\right)=0
\end{array}\right\}
$$

Where, the differential operator is $\nabla^{2}=\frac{\partial^{2}}{\partial \alpha^{2}}+\frac{\partial^{2}}{\partial \beta^{2}}$.

\section{The solution of free vibration eigenfrequency}

The solution of balance differential equations of free vibration can be solved by mixed method. Supposed circumferential load, that is $p_{1}=p_{2}=0$, the internal force function $\varphi=\varphi(\alpha, \beta)$ is brought in, and supposed,

$$
N_{1}=\frac{\partial^{2} \varphi}{\partial \beta^{2}}, N_{2}=\frac{\partial^{2} \varphi}{\partial \alpha^{2}}, N_{12}=-\frac{\partial^{2} \varphi}{\partial \alpha \partial \beta}
$$

The equations (2.1) and the equations (1.5) are brought in the third equation of the equations (1.2). The normal 
shell vibrates freely by the force of inertia. The normal free vibration balance differential equations of the cylindrical shell,

$$
\left.\begin{array}{l}
D \nabla^{4} w+\frac{1}{R} \frac{\partial^{2} \varphi}{\partial x^{2}}-m \frac{\partial^{2} w}{\partial t^{2}}=0 \\
\frac{1}{E h} \nabla^{4} \varphi-\frac{1}{R} \frac{\partial^{2} w}{\partial x^{2}}=0
\end{array}\right\}
$$

Dimensionless coordinate is brought in, $\xi=\alpha / R, \eta=\beta / R$, the equations (2.2) evolved to:

$$
\left.\begin{array}{l}
D \nabla^{4} w+R \frac{\partial^{2} \varphi}{\partial \xi^{2}}-m R^{4} \frac{\partial^{2} w}{\partial t^{2}}=0 \\
\frac{1}{E h} \nabla^{4} \varphi-R \frac{\partial^{2} w}{\partial \xi^{2}}=0
\end{array}\right\}
$$

Both of the two ends are supported, and the boundary conditions are,

$$
(w)_{\alpha=0}=0,(w)_{\alpha=l}=0,\left(M_{1}\right)_{\alpha=0}=0,\left(M_{1}\right)_{\alpha=l}=0
$$

Supposed the expressions of the internal force function $\varphi$ and the deflection $w$ are,

$$
\left.\begin{array}{l}
\varphi=\sum_{a} \sum_{b} A_{a b} \sin \lambda_{a} \xi \sin \mu_{b} \eta \sin \omega_{a b} t \\
w=\sum_{a} \sum_{b} B_{a b} \sin \lambda_{a} \xi \sin \mu_{b} \eta \sin \omega_{a b} t
\end{array}\right\}
$$

The boundary conditions (2.4) are fulfiled in the equations (2.5), and the equations (2.5) are brought in the equations (2.3),

$$
\left.\begin{array}{c}
-D\left(\lambda_{a}^{2}+\mu_{b}^{2}\right)^{2} \sum_{a} \sum_{b} B_{a b} \sin \lambda_{a} \xi \sin \mu_{b} \eta \sin \omega_{a b} t-R \lambda_{a}^{2} \sum_{a} \sum_{b} A_{a b} \sin \lambda_{a} \xi \\
\sin \mu_{b} \eta \sin \omega_{a b} t+m R^{4} \omega^{2} \sum_{a} \sum_{b} B_{a b} \sin \lambda_{a} \xi \sin \mu_{b} \eta \sin \omega_{a b} t=0 \\
\frac{1}{E h}\left(\lambda_{a}^{2}+\mu_{b}^{2}\right)^{2} \sum_{a} \sum_{b} A_{a b} \sin \lambda_{a} \xi \sin \mu_{b} \eta \sin \omega_{a b} t \\
-R \lambda_{a}^{2} \sum_{a} \sum_{b} B_{a b} \sin \lambda_{a} \xi \sin \mu_{b} \eta \sin \omega_{a b} t=0
\end{array}\right\}
$$

Equations (2.6) are simplified, that is,

$$
\left.\begin{array}{l}
R \lambda_{a}^{2} A_{a b}+\left[D\left(\lambda_{a}^{2}+\mu_{b}^{2}\right)^{2}-m R^{4} \omega_{a b}{ }^{2}\right] B_{a b}=0 \\
\frac{1}{E h}\left(\lambda_{a}{ }^{2}+\mu_{b}{ }^{2}\right)^{2} A_{a b}-R \lambda_{a}^{2} B_{a b}=0
\end{array}\right\}
$$

If the coefficient determinants of $A_{a b}$ and $B_{a b}$ are zero, $\varphi$ and $w$ are not identical to zero, that is,

$$
\left|\begin{array}{cc}
R \lambda_{a}^{2} & D\left(\lambda_{a}^{2}+\mu_{b}^{2}\right)^{2}-m R^{4} \omega_{a b}{ }^{2} \\
\frac{1}{E h}\left(\lambda_{a}^{2}+\mu_{b}^{2}\right)^{2} & -R \lambda_{a}^{2}
\end{array}\right|=0
$$

then the eigenfrequency is,

$$
\omega_{a b}=\left\{\frac{1}{m R^{2}}\left[\frac{D}{R^{2}}\left(\lambda_{a}^{2}+\mu_{b}^{2}\right)^{2}+\frac{E h}{\left(\lambda_{a}^{2}+\mu_{b}^{2}\right)^{2}} \lambda_{a}^{4}\right]\right\}^{\frac{1}{2}}
$$

Supposed the expression, $\quad \lambda_{a}=\frac{a \pi R}{l}(a=1,2,3, \mathfrak{w})$

Got the minimum value of $\lambda_{a}$, that is, $\lambda_{1}=\pi R / l$, and supposed $d \omega_{a b} / d \mu_{b}=0$, that is,

$$
\frac{d \omega_{a b}}{d \mu_{b}}=\frac{\mu_{b}}{m R}\left\{\frac{1}{m}\left[\frac{D}{R^{2}}\left(\lambda_{a}{ }^{2}+\mu_{b}{ }^{2}\right)^{2}+\frac{E h}{\left(\lambda_{a}^{2}+\mu_{b}{ }^{2}\right)^{2}} \lambda_{a}{ }^{4}\right]\right\}^{-\frac{1}{2}}\left[\frac{2 D}{R^{2}}\left(\lambda_{a}{ }^{2}+\mu_{b}{ }^{2}\right)-\frac{2 E h \lambda_{a}^{4}}{\left(\lambda_{a}{ }^{2}+\mu_{b}{ }^{2}\right)^{3}}\right]
$$

That is,

$$
\frac{2 D}{R^{2}}\left(\lambda_{a}^{2}+\mu_{b}^{2}\right)-\frac{2 E h \lambda_{a}^{4}}{\left(\lambda_{a}^{2}+\mu_{b}^{2}\right)^{3}}=0 \text {, and } D=\frac{E h^{3}}{12\left(1-\mu^{2}\right)}, \text { then }
$$

The minimum value of $\mu_{b}$ is,

$$
\mu_{b}^{2}=\lambda_{a}\left(\sqrt[4]{\frac{12 R^{2}\left(1-\mu^{2}\right)}{h^{2}}}-\lambda_{a}\right)
$$




$$
\mu_{1}^{2}=\lambda_{1}\left(\sqrt[4]{\frac{12 R^{2}\left(1-\mu^{2}\right)}{h^{2}}}-\lambda_{1}\right)
$$

The minimum eigenfrequency can be computed by $\lambda_{1}$ and $\mu_{1}$, that is,

$$
\omega_{\min }=\left\{\frac{1}{m R^{2}}\left[\frac{D}{R^{2}}\left(\lambda_{1}^{2}+\mu_{1}^{2}\right)^{2}+\frac{E h}{\left(\lambda_{1}^{2}+\mu_{1}^{2}\right)^{2}} \lambda_{1}^{4}\right]\right\}^{\frac{1}{2}}
$$

\section{The analysis of a certain AUV shell and emulation by ANSYS}

The shell of AUV is made up of spherical shell, cylindrical shell, conical shells and other revolutionary shells by thread coupling, bolt coupling, wedge coupling and hoop coupling. All of them are rigid coupling. The radius of the cylindrical shell is $R=0.265$, the length of cylindrical shell is $L=4.0$, the thickness of shell is $h=0.005$, the elastic modulus is $E=7.47 \times 10^{10} \mathrm{pa}$, the Poisson's ratio is $\mu=0.36$, the unit area mass of the cylindrical shell middle surface is $m=9.781$.

The bending strength $D=\frac{E h^{3}}{12\left(1-\mu^{2}\right)}=\frac{7.47 \times 10^{10} \times 0.005^{3}}{12\left(1-0.36^{2}\right)}=893.986$

The minimum value of $\lambda_{a}$ is, $\lambda_{1}=\frac{\pi R}{l}=\frac{\pi \times 0.265}{4.0}=0.20813$, then $\lambda_{1}^{2}=0.04332$

$$
\begin{aligned}
\mu_{b}^{2}= & \lambda_{1}\left(\sqrt[4]{\frac{12 R^{2}\left(1-\mu^{2}\right)}{h^{2}}}-\lambda_{1}\right)=0.20813 \times\left(\sqrt[4]{\frac{12 \times 0.265^{2} \times\left(1-0.36^{2}\right)}{0.005^{2}}}-0.20813\right)=2.68062 \\
\omega^{2}= & \frac{1}{m R^{2}}\left[\frac{D}{R^{2}}\left(\lambda_{a}^{2}+\mu_{b}^{2}\right)^{2}+\frac{E h}{\left(\lambda_{a}^{2}+\mu_{b}^{2}\right)^{2}} \lambda_{a}^{4}\right]=\frac{1}{9.781 \times 0.265^{2}}\left[\frac{893.986}{0.265^{2}}(0.04332+2.68062)^{2}\right. \\
& \left.+\frac{7.47 \times 10^{10} \times 0.005}{(0.04332+2.68062)^{2}} \times 0.20813^{4}\right]=275035.75
\end{aligned}
$$

The minimum eigenfrequency is,

$$
\omega=\frac{\sqrt{275035.75}}{2 \pi}=83.47 \mathrm{~Hz}
$$

Emulation analysis of the cylindrical shell is operated using the ANSYS, and the minimum eigenfrequency is $84.77 \mathrm{~Hz}$.

\section{Conclusions}

Sum up, the error between the computational result and the emulational result of the minimum eigenfrequency is $1.53 \%$ The different eigenfrequency can be calculated by different $\lambda_{a}$ and $\mu_{b}$, the error between the computational result and the emulational result of the eigenfrequency is no more than $5 \%$. The method is effective in analyse and dynamical design of AUV weaken vibration and low noise structure.

\section{References}

P. Smithmaitrie \& H.S. Tzou. (2004). Micro-control actions of actuator patches laminated on hemispherical shells. Journal of Sound and Vibration. 277, pp. 157-164

J.H. Ding \& H.S. Tzou. (2004). Micro-electromechanics of sensor patches on free paraboloidal shell structronic systems Mechanical Systems and Signal Processing 18. pp. 367-380

Yang, Depin. (1988). Applied shell mechanics. The Hunan University Press.

Xu, Zhilun. (1982). Elastomechanics . The High Education Press.

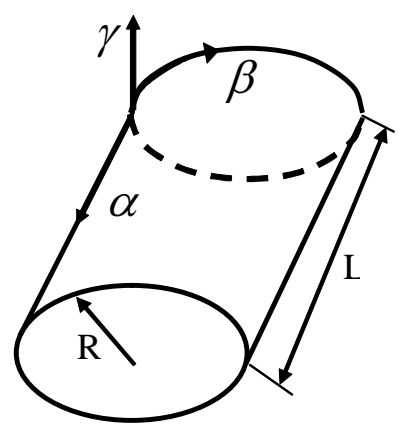

Figure 1. The coordinate

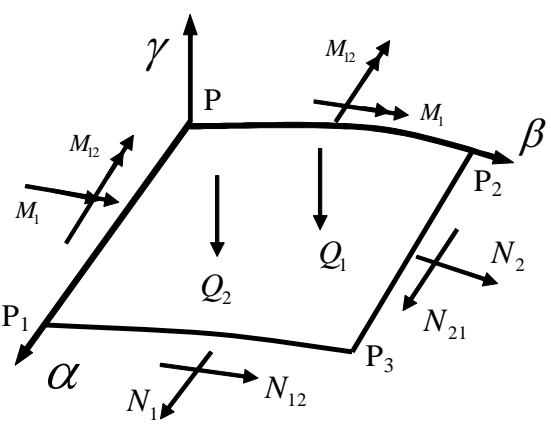

Figure 2. The internal forces 